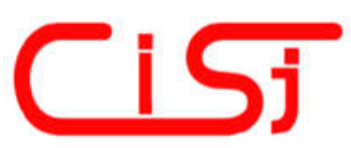

\title{
COST EFFECTIVE ENERGY EFFICIENT SCHEME FOR MOBILE ADHOC NETWORK
}

\author{
Rathish Radhakrishnan ${ }^{1)}$, Karpagavadivu Karuppusamy ${ }^{2)}$ \\ 1) Department of Electronics and Communication Engineering, United Institute of Technology, Coimbatore, 641020 , \\ India, r.rathish87@gmail.com \\ 2) Department of Information Technology, Bannari Amman Institute of Technology, Sathyamangalam, 638 401, India, \\ karpagavadivu@bitsathy.ac.in
}

Paper history:

Received 23 November 2018

Received in revised form 19 December 2019

Accepted 21 February 2020

Available online 31 March 2020

Keywords:

Clusters;

MANET;

Gateway;

LEACH;

Broadcasting;

Energy Efficiency;

Power;

REQ;

RES Consumption;

Routing.

\begin{abstract}
Clustering is defined as grouping of nodes based on their energy levels. In each cluster, a gateway node is set depending on the node that has the highest energy level. This phenomenon is majorly implemented using LEACH protocol. LEACH is abbreviated as Low Energy Adaptive Clustering Hierarchy. It is a time division-based Media Access Control (MAC) protocol. In order to make it energy efficient a new protocol is defined to hold the sensors as long as possible. It balances the network load in minimal time. A more energy efficient node transfer is possible by means of the above protocol which attains the high residual energy. The major advantage of implementing this protocol is to reduce the energy and power consumption that is needed to create and maintain the cluster. In Mobile Ad-hoc Network (MANET), the vast consumption of power can be literally minimized by utilizing this clustering scheme. The simulation of our protocol is carried out in Network Simulator-2.
\end{abstract}

Copyright (C) Research Institute for Intelligent Computer Systems, 2020. All rights reserved.

\section{INTRODUCTION}

There are so many variations in forming a clustering algorithm. The ultimate use of clusters is vibrantly found in data mining, data analysis, pattern study and graphical interpretation. Each algorithm tends to differ significantly based on its own characteristics. Therefore clustering holds multiple objective of balancing power utilization [1].

The centroid model of clustering (for example, kmeans algorithm) plays the part of single mean vector. The discrepancy between the clusters is calculated by considering its average value [2]. In the k-means clustering algorithm the processing of signals as well as quantization is extensively done. For example, in $\mathrm{n}$ number of observations ( $\mathrm{x} 1$, $x 2, \ldots . . x n)$, this clustering algorithm targets to segregate the $\mathrm{n}$ observations into $(k \leq n)$. Thus, it is observed that the bisecting $\mathrm{k}$-means are more preferable than the regular k-mean [3]. Whereas the distribution model and density model of clustering personify statistical distribution and cluster establishment in dense region.
The density of the clusters as well as the header node or gateway node is determined and initialized based on their energy levels. One of the simplest protocol that deals with the clusters and gateway formation is Low Energy Adaptive Clustering Hierarchy (LEACH) [4]. This Time Division based protocol transmits the data packets from the source to destination through a particular gateway node which is equipped with the energy of a particular node in the cluster. The gateway formation is a round-up process in which every node tends to participate. At the end of this process the node with the maximum energy level will be considered as the gateway node. The node that was said to be the gateway at the end of a round process cannot be assigned as a gateway node for the second time.

In the proposed method, the gateway of a particular cluster that has the source node identifies the gateway of another cluster that acts as a bridge between the source node and the cluster that has the destination node. Each cluster head holds a particular schedule to transmit the data. The major 
reason for setting up a gateway node or cluster head is that it effectively reduces the power consumption.

This paper deals with the transmission of data based on the acknowledgement of the destination which is denoted as RES. This response is generated only when the source node transmits a requisition REQ for sending a data packet. This REQ and RES messages will be transferred with the help of the gateway node. The section II of this paper deals with the work done according to the proposed algorithm. The section III of this paper is related to the existing concept of flooding technique. The section IV projects our proposed algorithm in detail. In section $\mathrm{V}$, the output and performance of our research has been established.

\section{RELATED WORK}

Atif A. Alghamdi, Robert J. Pooley and Peter J. B. King proposed a flooding concept in which the broadcasting of each node is determined as every node tends to retransmit at least once. They established an energy efficient forwarding scheme that utilizes the one-hop neighboring radios. Here positioning and location is not needed. Even though they combine different techniques that collaborate to conserve energy, the major limitation of their work is congestion occurrence and power consumption [5].

A. Ahmad, M.A. Pasha, Z. Ahmad, S. Masud and A. Sikora designed an Energy Efficient Sensor Network Routing (EESNR) Protocol for Large Distributed Environmental Monitoring Applications which resolves the issues encountered by other traditional hierarchical multi-hop routing protocols. This algorithm increases the lifespan of each WSN node which in turn enhances the lifespan of the entire network. But due to the increase in the network lifespan, energy consumption is higher and it also encounters the congestion problem [6].

Mythili S. and Madhiya E. conducted an analysis on clustering algorithms and predicted the same advantages and shortcomings. Their partitional algorithm obtains a single partition instead of clustering structure. Proximity plays a major role in their proposed concept. Their analysis revealed that the clustering algorithms tend to lose information and do not find an appropriate number of clusters [7].

Michael Steinbach, GeorgeKarypis and Vipin Kumar presented an experimental study of some clustering techniques. They finally concluded that bisecting k-means algorithm is far better than regular $\mathrm{k}$-mean technique but it suffers from the drawback that depends on the initial number of cluster ' $k$ ' given by the user [8].
H. Jeong and Y. Yoo made a note on flooding process in which all the nodes transmit simultaneously. This results in broadcasting problem which leads to a serious case. Therefore the network resources become severely wasted. In order to minimize this problem, the transmission of data from a neighbor to another has been established. A back off delay scheme is adopted to avoid collision of nodes which results in congestion/traffic, but this method lacks flexibility and moreover it does not react to the network failure [9].

C. R. Rathish and A. Rajaram proposed a system which increases the lifetime of the sensors by establishing a multiple hop network that protectively transmits the received messages. It also finds out the best possible path for routing by considering the various parameters such as hop distance, delay and the like. Moreover, minimal time load balancing is also achieved in this system. The proposed algorithm SICRA utilizes less power and provides better connectivity [10].

A probabilistic broadcast scheme was brought into practice by D.G. Reina, S.L. Toral, P. Johnson, and F. Barrero. This scheme plays an important role in the performance of the network such as low overhead, balanced energy consumption and robustness. However, it encounters other problems such as collision, contention and message duplication [11].

A. Al-Qawasmeh and S. Bettaye pointed out that even though there were several flooding techniques available for MANETs, most of them assume that every node in the network has the same transmission range. Therefore, those techniques show poor performance when the nodes in the network have different transmission ranges. So they proposed a new flooding technique to support nodes with different transmission ranges in MANET. Thus, when a node receives a packet, it avoids unnecessary retransmission by checking whether the transmission has occurred in all the nodes. However, the energy consumption is high and also suffers from interference problems due to high network coverage [12].

C. R. Lin and M. Gerla made a network architecture where the clusters are independently controlled and dynamically configured as the nodes move. Thus, it leads to spatial reuse and controls the reservation of bandwidth [13].

F. Xiangning and S. Yulin made a study on the performance of multi-hop leach protocol as it improves the choice of selecting the cluster head based on the node which has more residual energy [14].

An analysis was done by V. Katiyar, N. Chand, G. C. Gautam and A. Kumar on the major deficiency that degrades the performance of a protocol, i.e., the 
presence of large as well as small clusters in the same network at the same time. A new energy efficiency protocol that eliminates the above problem by forming a 'far-zone' has been established. Far-zone is nothing but a group of sensor nodes which is placed at a location where the energy is less than their threshold [15].

C R Rathish and A Rajaram presented a load balanced routing algorithm based on hierarchy of the nodes in the network. The problem of congestion in the network is eliminated by performing the load balancing and also path stability is provided depending on the link bandwidth, cost, quality and load balancing. It provides energy efficient routing by computing the residual energy of the nodes [16].

After analyzing the critical issue in designing a WSN protocol, (that is, the energy consumed while transferring data from the sensor node) J. Xu, N. Jin, X. Lou, T. Peng, Q. Zhou and Y. Chen proposed a new algorithm named Improvement of LEACH Protocol for WSN that enhances the original way of selecting the cluster head [17].

C.Y. Fu, Z. Jiang, W. Wei and A. Wei proposed a new protocol called LEACH-TLCH which is designed to balance the energy consumption of the entire network. Thus, it leads to the increase in energy efficiency and lifetime of the network more than the LEACH protocol but suffers from delay and network overhead [18].

M. J. Handy, M. Haase and Timmermann majorly focused on the power consumption of WSN and they increased the network lifetime by about $30 \%$. However, the main drawback of this method is the non-automatic cluster head selection which reduces the efficiency of the overall system [19].

S. Corson and J. Macker described the characteristics of MANET based on traditional and hard wired packet networks. This network host supports 'roaming' where the roaming host can be connected through various fixed address domain space [20].

A. Al-Maashri and M. Ould-Khaoua examined the MANET routing protocols such as DSR, AODV, OLSR based on their PDR, overhead, throughput and delay [21].

O. Younis and S. Fahmy proposed a novel distributed clustering approach for long-lived ad-hoc networks. HEED periodically selects cluster head according to the node residual energy and node proximity. However, the random selection of the cluster head results in network overhead and also consumes more energy [22].

\section{EXISTING ALGORITHM}

Atif A. Alghamdi, Robert J. Pooley and Peter J. B. King proposed a design based on the flooding technique. They classified the nodes as gateway nodes and non-gateway nodes based on their transmission power level pt [5]. According to the proposed method, the nodes that have the same $\mathrm{pt}$ level are mostly located within the same group. Thus, in this case the neighbor nodes may tend to receive the same broadcast packets. The pt level hence is utilized in order to obtain more efficient and reliable flooding. This method suffered from two major drawbacks i.e., energy reservation which is the main resource of the nodes and increase in the congestion and overheads that degrades the performance of the network..

The various details provided in this article include: i) In order to determine whether the node is a gateway or not, the exchange of packets precisely the control packets takes place. ii) In AODV protocol, the route discovery has been analyzed i.e., when a source node is ready to transmit a packet but the route is not available, the source node will broadcast a request RREQ to find a path. iii) When the RREQ packet finds an intended destination that has a route towards the destination node, the data packets organize a unicast reply RREP along the reverse path established during the route discovery.

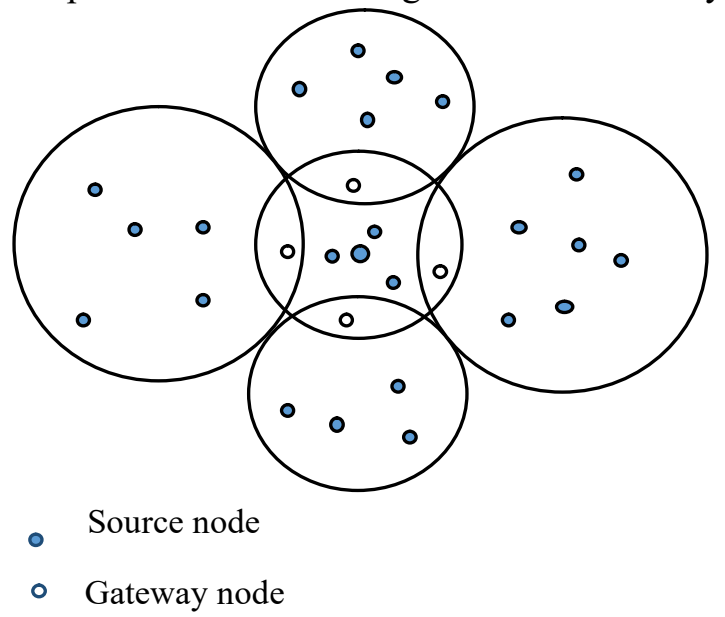

Figure 1 -Existing gateway forwarding

Table 1. Neighbour table of non-gateway nodes

\begin{tabular}{|c|c|}
\hline Neighbour & Pt level \\
\hline A & Low \\
\hline B & Low \\
\hline C & Low \\
\hline D & Low \\
\hline
\end{tabular}

In the existing method they have combined different techniques that collaborate to provide energy conservation, but the major limitation of this existing work is the congestion occurrence in the network which reduces the overall efficiency of the system and also utilizes more power [5]. 


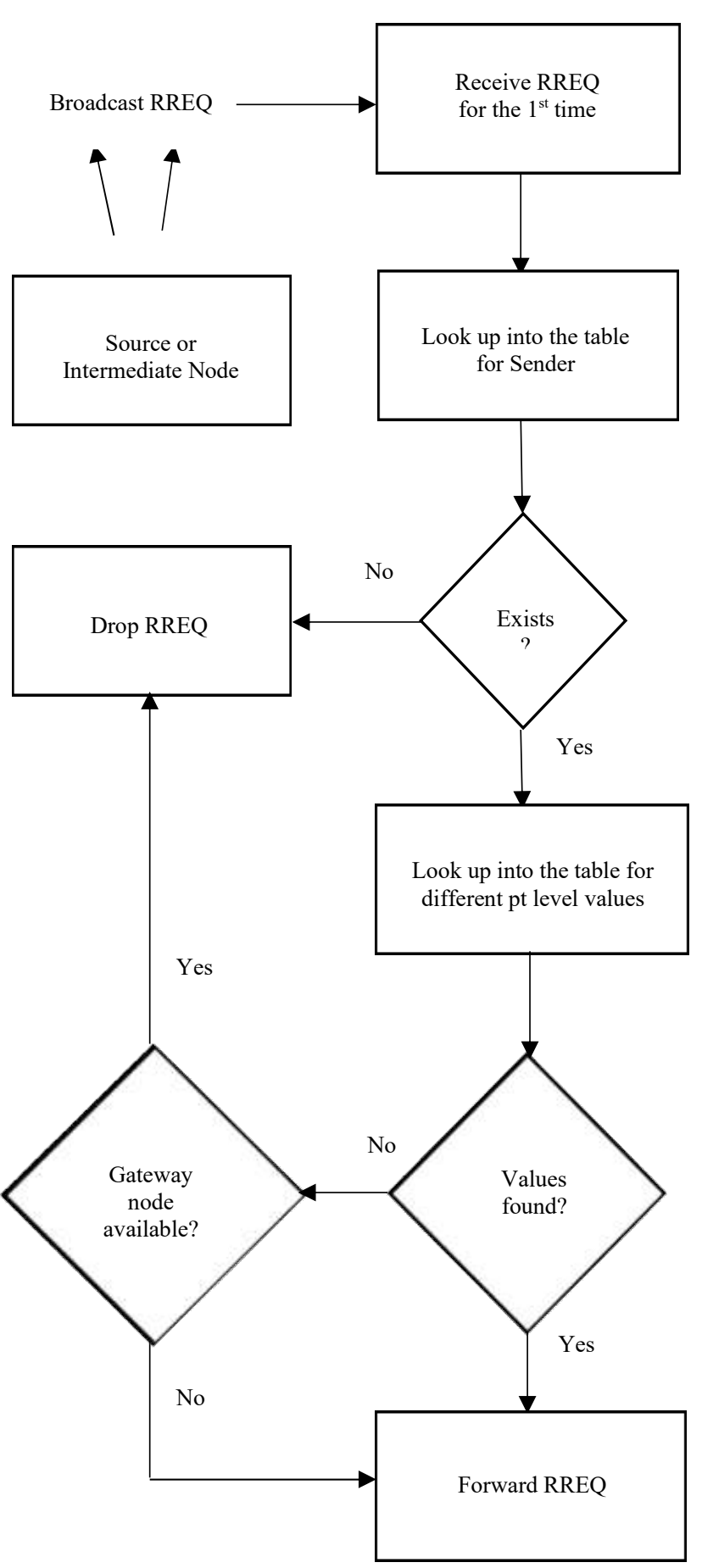

Figure 2 - Flowchart of existing algorithm

\section{PROPOSED ALGORITHM}

In the previous methodology, an on-demand distance routing algorithm was implemented. The major consequence of the protocol is that the route is created only on the basis of the requisition of the source node in order to transmit the data packets. In our proposed algorithm we use Low Energy Adaptive Clustering Hierarchy (LEACH) protocol which highly concentrates on energy optimization and minimizes the congestion occurrence in the network that obviously leads to the improvement of the network's lifetime.

Initially in our process, the mobile ad-hoc network (MANET) is created which is involved in the broadcasting of the data packets. The broadcasting occurs as the updating of neighborhood nodes is completed. The route of the gateway node that is the route discovery occurs by reducing the routing overhead. We mainly concentrate on energy conservation and reduction of power consumption with respect to the transmission cost.

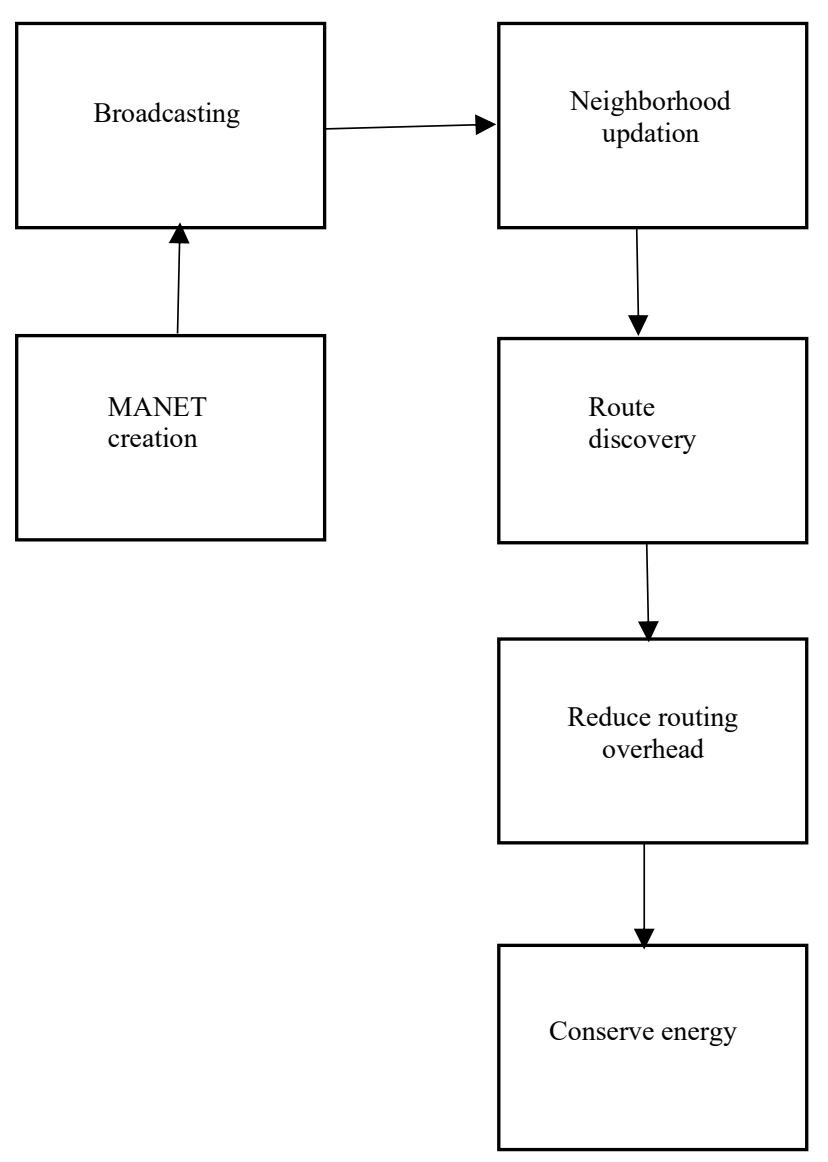

Figure 3 - Block diagram

A cluster with a gateway node plays a major role in processing the data packets. The gateway is identified by considering the node that has the highest residual energy level. The nodes with similar energy levels are clustered together and hence in each cluster a gateway will be initialized. While transmitting the data packets the gateway acts as an intermediate bridge that connects the sender node and the receiver node. By doing this the load optimization could be done.

MANET (Mobile Ad-hoc Network) is an infrastructure-less wireless network so it does not require any cental administration. These nodes in the network do not only work as hosts but also act as 
routers in order to communicate with each other through packet radio.

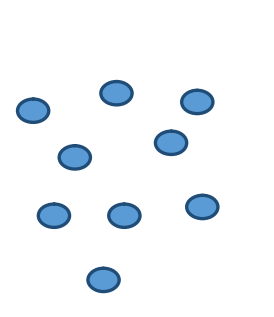

$\mathrm{GW}$

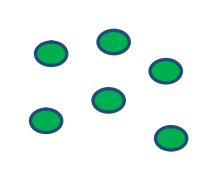

GW
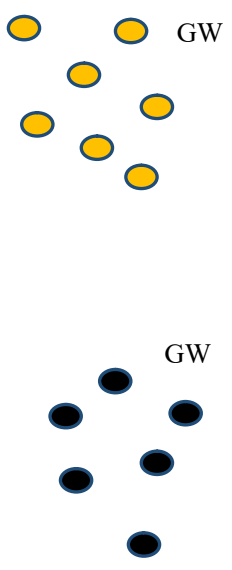

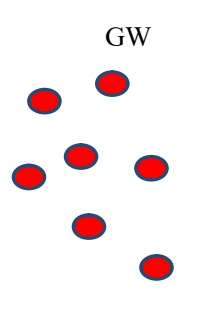

Figure 4 - Representation of clusters and gatewav

Since these networks are not directly connected to a power supply, the replacement of battery is a major challenge. The energy consumption is given high priority in case of power management techniques. Although many routing protocols are already in existence, LEACH is considered as the most popular routing protocol that uses cluster based algorithm to minimize the power consumption. LEACH has properties such as multi-hop routing, distributed cluster formation, reducing global communication and random rotation of cluster heads.

In the Network Simulator-2, the topology, scheduling algorithm, transportation protocol and routing can be simulated. This tool helps us to study the basic network components. In our proposed concept, we use NS - 2 for simulation with a virtual OS mechanism named FEDORA. We have set the coding based on the user interface effectiveness such that the sender node and receiver node are intruded manually. At the initial stage, when the nodes (42) were created, they were shown in black. Once they were defined they turned to green. This indicates that the definition of nodes was successful.

The round-up process of the gateway selection begins as the energy of each and every node is identified. Thus, the gateway will be assigned to the node having the maximal energy level. Similarly, each cluster takes up this process of selecting their own gateway. The nodes with more or less similar residual energy level were grouped together as clusters. The algorithm that indicates the working of our protocol includes

STEP 1: Create a sensor network model.

STEP 2:Assign initial energy to the nodes of the network.
STEP 3: Divide the network into clusters.

STEP 4: Check the energy levels of the nodes. If Energy $>$ max, select the node as cluster head Else

Leave it as a cluster member

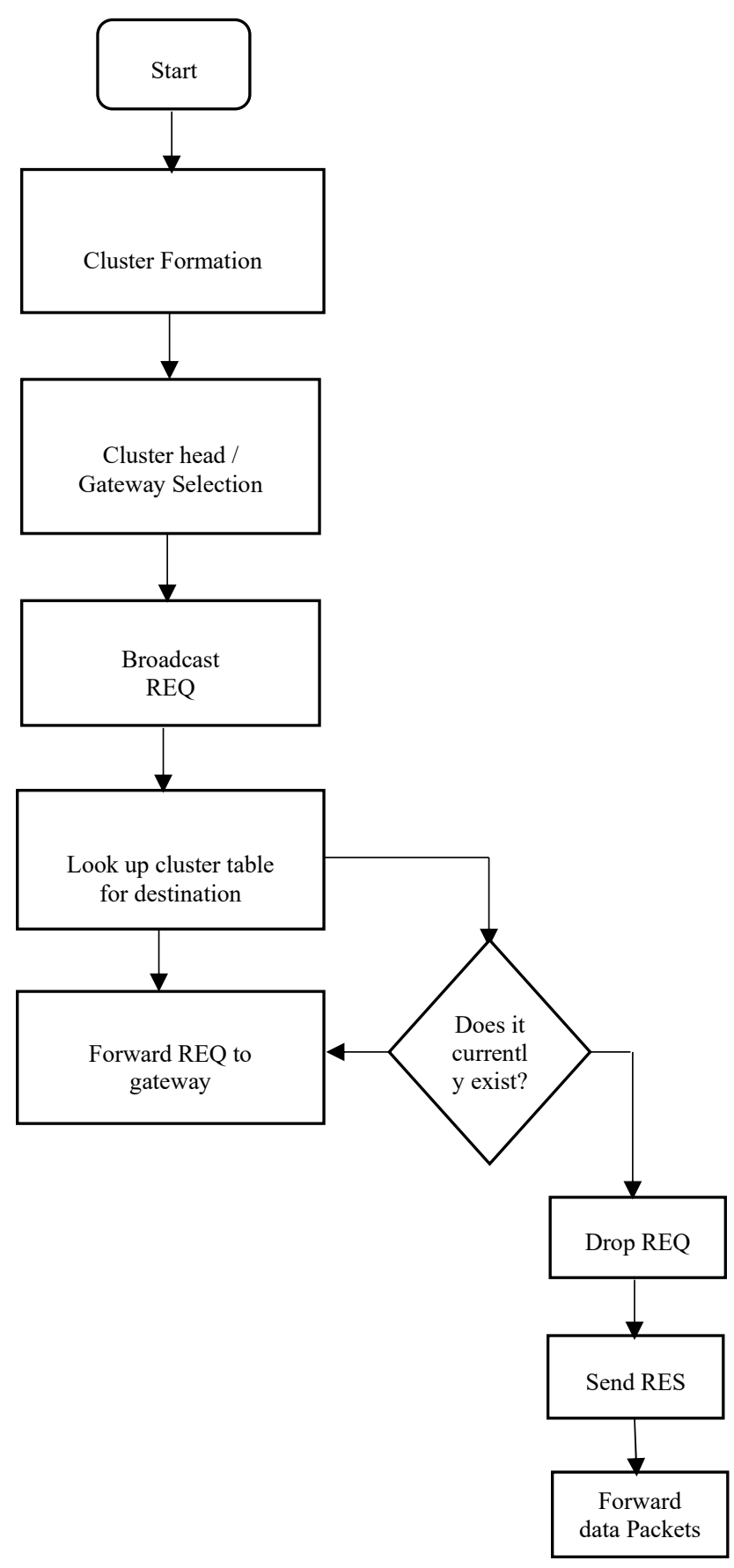

Figure 5 - Flow of LEACH

\section{SENDING REQ:}

STEP 1: If the node receives request REQ for the $1^{\text {st }}$ time, it checks the routing table for its sender.

STEP 2: If REQ sender currently exists then the node determines whether it is a gateway or not. STEP 3: If destination is within its cluster 


\section{Drop REQ \\ Else \\ Forward to gateway}

\section{SENDING RES:}

STEP 1: If the cluster head receives REQ, it checks the neighbour table.

STEP 2: If destination currently exists

Drop REQ and send RES in a fresh route in which it received REQ

\section{Else \\ Forward REQ}

Our paper deals with the above algorithm in which the execution of the output is carried out. After the initialization of nodes a request REQ will be transmitted by the sender node requesting for a data transmission with a corresponding receiver node. The node that carries REQ checks the neighbour table for a suitable gateway needed for their transmission ensuring that the data transfer occurs only through it. Once the gateway is determined, it redirects the REQ to the destination. Thus, the receiver node responds to the sender node whether it is ready to receive the data packet or not through RES. This is known as the response of the destination node. Likewise the data packet transmission occurs as a continuous process.

\subsection{DATA TRANSMISSION PROCESS}

In the proposed Cost Effective Energy Efficient Scheme (CEEES), the source and the destination nodes are selected which aids in the creation of the nodes. Once the nodes have been created they are initialized and based on their energy levels they are formed into clusters. After this, the gateway nodes are assigned and then the data transmission takes place from the source to the destination node.

\begin{tabular}{|c|}
\hline 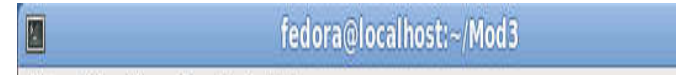 \\
\hline File Edit View Teminal Help \\
\hline 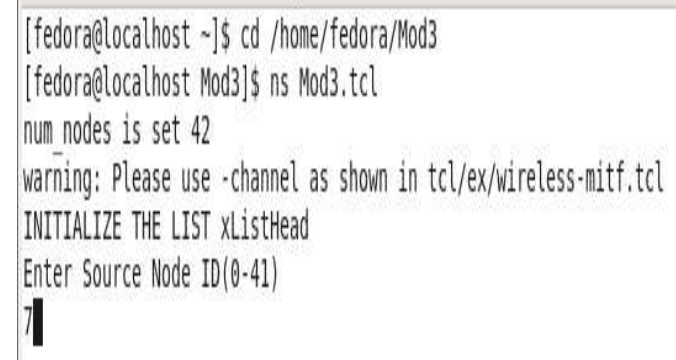 \\
\hline
\end{tabular}

\section{Figure 6 - Source node selection}

Node 7 is selected as the source node from which the data packet will be transmitted.

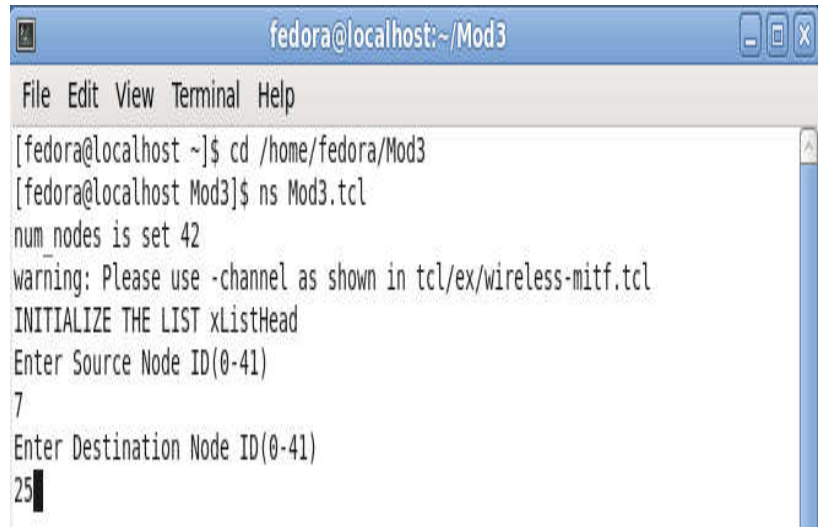

Figure 7 - Destination node selection

Node 25 is selected as the destination node where the data packet sent from source node will be received.

Here, the source and destination nodes are selected manually which can be changed according to the requirement.

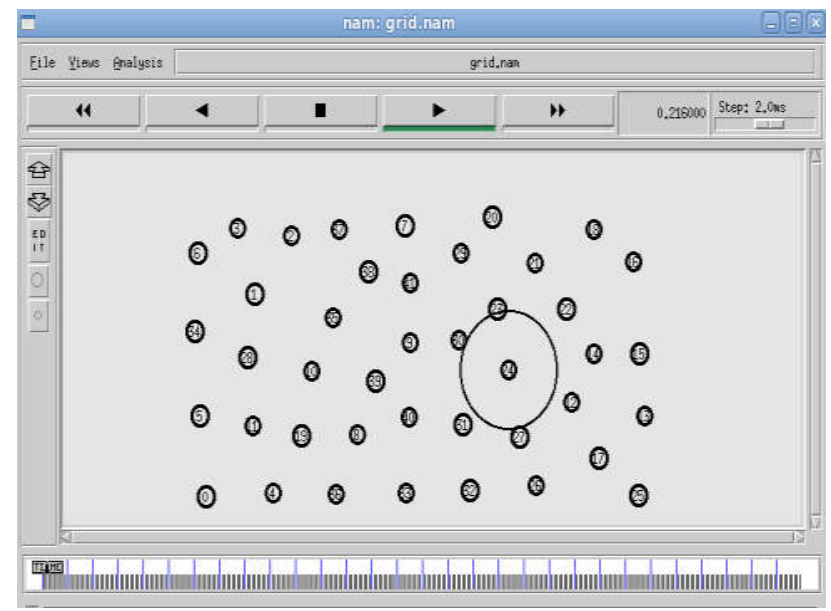

Figure 8 - Node Creation

In the proposed system, in order to avoid congestion 42 nodes have been created using the commands in NS-2 Simulator.

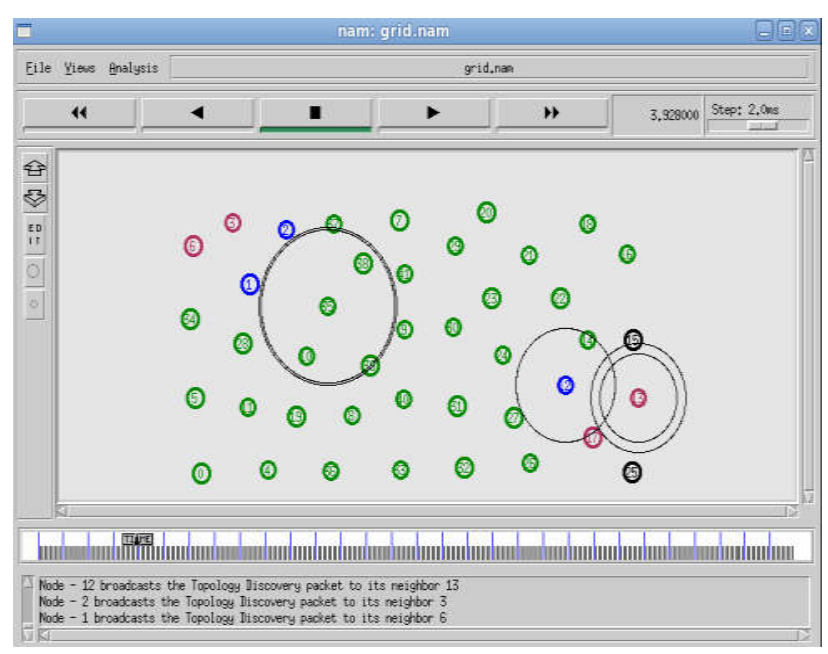

Figure 9 - Node Initialization 
Once the nodes are created their initialization process begins. The blue nodes denote the sender nodes that transmit the data to the receiver whereas the pink nodes denote the receiver node that receives the transmitted data from the sender.

The black nodes denotes the uninitialized nodes. And finally the green nodes stand for the indication of declared nodes.

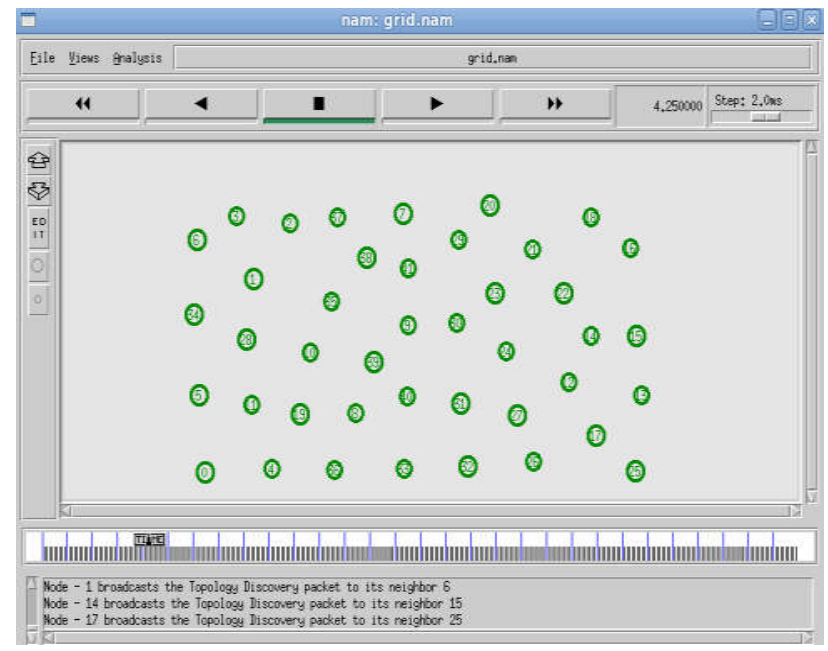

Figure 10 - Initialized Nodes

Once the initialization of all the nodes is completed they will be represented in green.

Clusters are formed based on the energy levels and thus for a clear identification they are denoted by various colours.

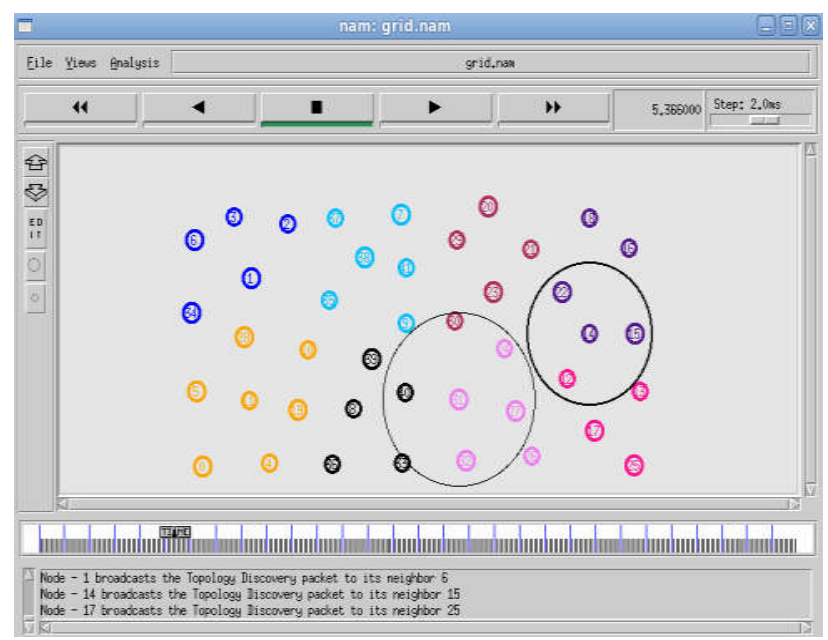

Figure 11 - Cluster Formation

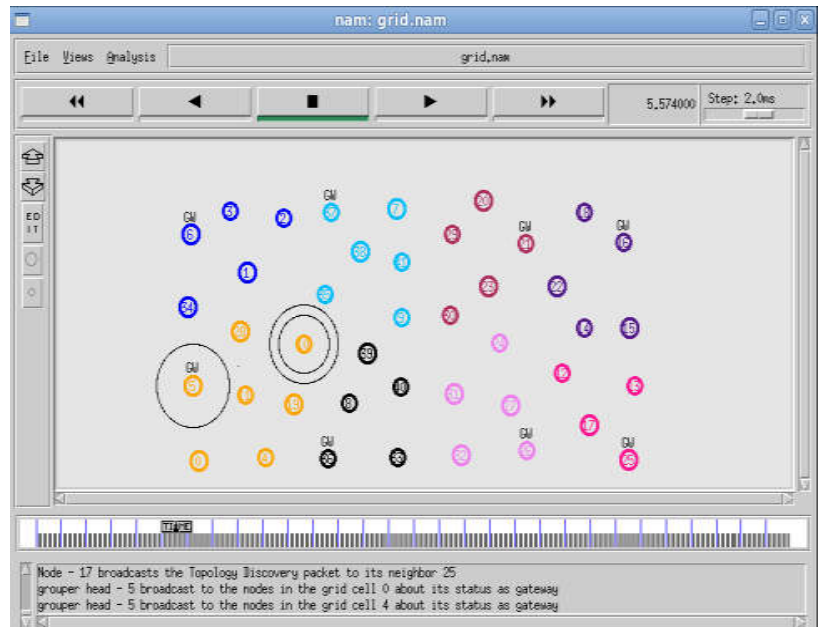

Figure 12 - Assignment of gateways

The nodes with the indication GW represent the gateway nodes.

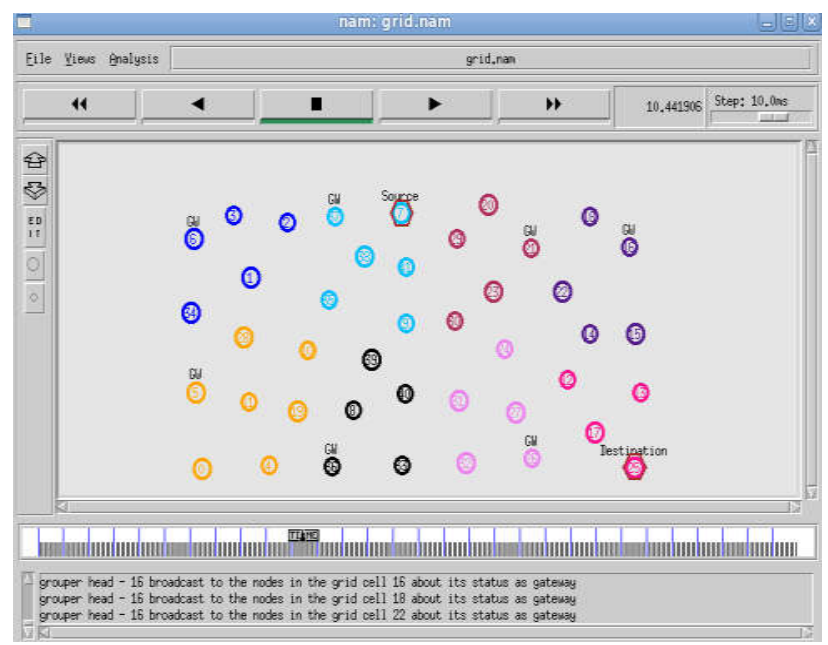

Figure 13 - Data transfer between nodes

As the nodes 7 and 25 represent the source and destination node respectively, at the output.1., the nodes numbered 7 and 25 were named as source and destination.

\section{PERFORMANCE ANALYSIS}

The Network Simulator (NS-2) is used to reproduce the proposed scheme. NS-2 is one of the efficient simulation tools used in Wireless Sensor Networks. The constructed technique is programmed by writing the $\mathrm{C}++$ program. Moreover, the tool supports establishing the procedure logically. In our simulation 42 mobile nodes are used. Our simulation settings and parameters are summarized in Table 2. 
Table.2. Specification analysis

\begin{tabular}{|c|c|}
\hline Specification & Numbers \\
\hline No. of Nodes & 42 \\
\hline No. of packets & 4920 \\
\hline Highest antenna & 1.5 \\
\hline Dist CST & 1143.2 \\
\hline No. of grid cells & 8 \\
\hline Protocol & LEACH \\
\hline
\end{tabular}

\subsection{RESIDUAL ENERGY}

Residual energy is the energy level that can be constructed either by direct approach or prediction based approach

$$
\Delta E=\sum_{i=1}^{k} \Delta e i,
$$

where $\Delta \mathrm{E}$ represents the amount of energy consumption.

$\Delta$ ei represents the energy spent in a single state by the node.

Figure 14 shows the result of average residual energy for varying the time from 5 to $25 \mathrm{~ms}$. From the result it is observed that the proposed Cost Effective Energy Efficient Scheme (CEEES) has lower enery usage when compared to the existing Energy Efficient Adaptive Forwarding Scheme (EEAFS).

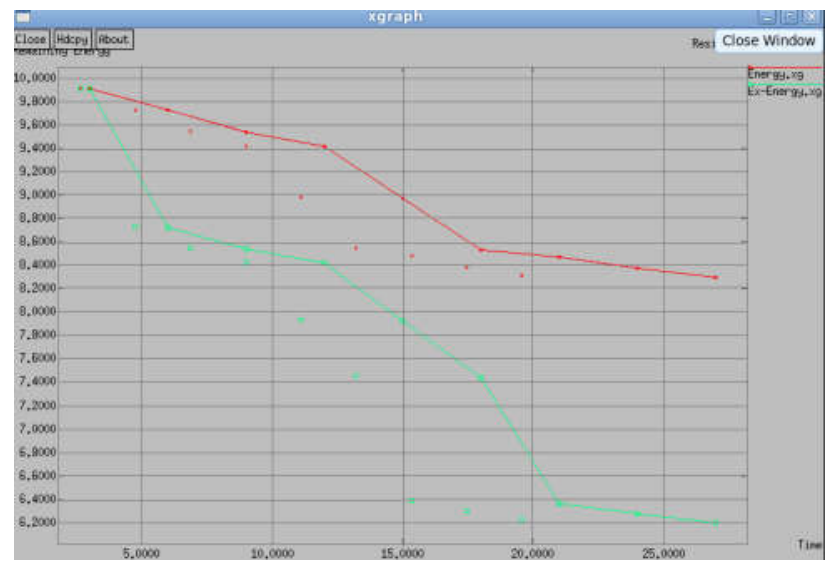

Figure 14 - Time Vs Remaining energy

Table.3. Remaining energy

\begin{tabular}{|c|c|c|}
\hline \multirow{2}{*}{ Time (ms) } & \multicolumn{2}{|c|}{ Remaining Energy (joules) } \\
\cline { 2 - 3 } & EEAFS & CEEES \\
\hline 5 & 9 & 9.7 \\
\hline 10 & 8.5 & 9.5 \\
\hline 15 & 7.8 & 8.9 \\
\hline 20 & 6.6 & 8.4 \\
\hline 25 & 6.3 & 8.2 \\
\hline
\end{tabular}

Thus, the table shows that the energy consumption of the proposed system is lower than that of the existing system.

\subsection{PDR (PACKET DELIVERY RATIO)}

It is defined as the ratio of data packets that is being sent by the sender and received by the receiver. If $\mathrm{x} 1$ is the data packets sent by the sender and $\mathrm{x} 2$ is the data packet received by the receiver, then it can be mathematically given as

$$
P D R=\frac{x 1}{x 2} .
$$

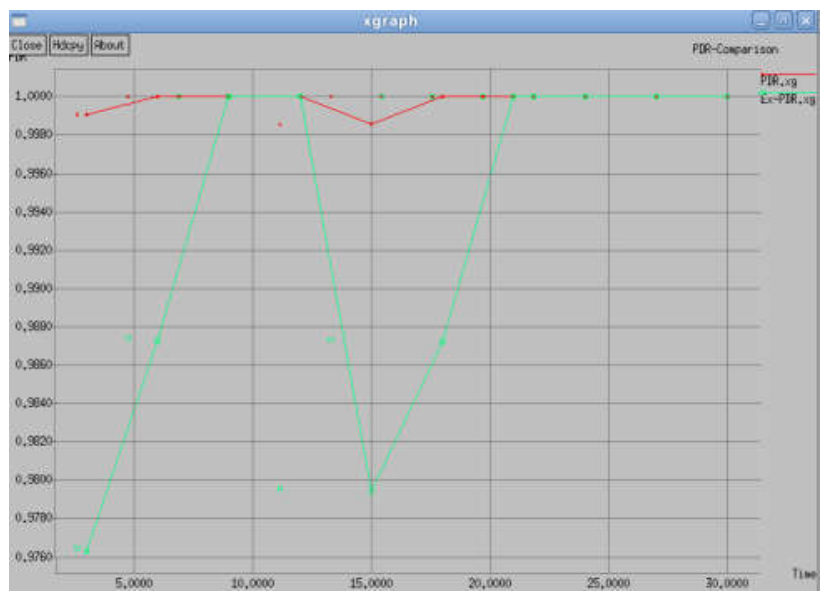

Figure 15 - Time Vs PDR

Fig. 15 represents the packet delivery ratio comparison for the existing EEAFS and proposed CEEES techniques. This noticeably indicates that the amount of packets succeeded by the proposed system is maximum when compared to the existing one.

Table 4. Packet delivery ratio.

\begin{tabular}{|c|c|c|}
\hline \multirow{2}{*}{ Time (ms) } & \multicolumn{2}{|c|}{ Packet Delivery Ratio } \\
\cline { 2 - 3 } & EEAFS & CEEES \\
\hline 5 & 0.9840 & 0.9996 \\
\hline 10 & 1 & 1 \\
\hline 15 & 0.9796 & 0.9984 \\
\hline 20 & 0.9960 & 1 \\
\hline 25 & 1 & 1 \\
\hline
\end{tabular}

Due to the table it is inferred that the Packet Delivery Ratio of the proposed CEEES is found to be higher than the existing EEAFS. 


\section{CONCLUSION}

In the proposed CEEES algorithm, our main concentration was the reduction of power consumption and enhancement of load balancing, load optimization and obtaining higher energy efficiency. We have finally concluded that our 1st parameter which is the packet delivery ratio (PDR) shows a result in attaining $96 \%$ of effective communication in the form of data receiving base. Our 2nd result is based on concluding that maximum nodes will live till the end showing the effectiveness of our system. This is actually obtained by taking a random selection of nodes. Finally, the throughput of the network turns better as it reduces the traffic occurrence. The major disadvantage of LEACH is that the nodes in the cluster tend to die when the cluster head dies. In our future perspective, the uneven cluster separation and distribution can be done without degrading the performance of the entire network.

\section{REFERENCES}

[1] S.S. Ghuman, "Clustering techniques - a review," International Journal of Computer Science and Mobile Computing, vol. 5, issue 5, pp. 524-530, May 2016.

[2] P. Berkhin, "A Survey of Clustering Data Mining Techniques," in: Kogan J., Nicholas C., Teboulle M. (eds) Grouping Multidimensional Data. Springer, Berlin, Heidelberg, 2006, pp. 25-71.

[3] N. Nasser, C. Arboleda, M. Liliana, N. Nasser, C. Arboleda, M. Liliana, "Comparison of Clustering Algorithms and Protocols for Wireless Sensor Networks," Proceedings of the Canadian Conference on Electrical and Computer Engineering, 2006, pp. 1787-1792.

[4] W. Akkari, B. Bouhdid, A. Belghith, "LEATCH: low energy adaptive tier clustering hierarchy," Proceedings of the 6th International Conference on Ambient Systems, Networks and Technologies, 2015, pp. 365372.

[5] A.A. Alghamdi, R.J. Pooley, P.J.B. King, "Energy-efficient adaptive forwarding scheme for MANETs," Proceedings of the 2016 Wireless Days (WD), 2016, pp. 1-7.

[6] A. Ahmad, M.A. Pasha, Z. Ahmad, S. Masud, A. Sikora, "Energy Efficient sensor network routing (EESNR) protocol for large distributed environmental monitoring applications," Proceedings of the 9th IEEE International Conference on Intelligent Data Acquisition and Advanced Computing Systems: Technology and Applications (IDAACS2017), 20-23 September 2017, Bucharest, Romania, pp. 740-745.
[7] S. Mythili, E. Madhiya, "An analysis on clustering algorithms in data mining," International Journal of Computer Science and Mobile Computing, vol. 3, issue 1, pp. 334-340, January 2014.

[8] M. Steinbach, G. Karypis, V. Kumar, "A comparison of document clustering techniques," Proceedings of the KDD Workshop on Text Mining, 2000, pp. 1-20.

[9] H. Jeong, H. Jeong, Y. Yoo, "Dynamic probabilistic flooding algorithm based on neighbor information in wireless sensor networks," Proceedings of the International Conference on Information Networking (ICOIN), 2012, pp. 340-345.

[10] C.R. Rathish, A. Rajaram, "Sweeping inclusive connectivity based routing in wireless sensor networks," ARPN Journal of Engineering and Applied Sciences, vol. 13, no. 5, pp. 1752-1760, March 2018.

[11] D.G. Reina, S.L. Toral, P. Johnson, F. Barrero, "A survey on probabilistic broadcast schemes for wireless ad-hoc networks," $A d$ Hoc Networks, vol. 25, pp. 263-292, February 2015.

[12] A. Al-Qawasmeh, S. Bettaye, "A proactive distance-based flooding technique for MANETs with heterogeneous radio ranges," Proceedings of the 33rd IEEE Conference on Local Computer Networks (LCN), 2008, vol. 1, pp. 1-21.

[13] C.R. Lin, M. Gerla, "Adaptive clustering for mobile wireless networks," IEEE Journal on Selected Areas in Communications, vol. 15, issue 7, pp. 1265-1275, September 1997.

[14] F. Xiangning, S. Yulin, "Improvement on LEACH protocol of wireless sensor networks," Proceedings of the International Conference on Sensor Technologies and Applications (SENSORCOMM'2007), October 2007, pp. 260-264.

[15] V. Katiyar, N. Chand, G. C. Gautam, A. Kumar, "Improvement in LEACH protocol for large scale wireless sensor networks," Proceedings of the International Conference on Emerging Trends in Electrical and Computer Technology (ICETECT), 2011, pp. 1070-1075.

[16] C.R. Rathish and A. Rajaram, "Hierarchical Load Balanced Routing Protocol for Wireless Sensor Networks," International Journal of Applied Engineering Research, vol. 10, issue 7, pp. 16521-16534, 2015.

[17] J. Xu, N. Jin, X. Lou, T. Peng, Q. Zhou, Y. Chen, "Improvement of LEACH protocol for WSN," Proceedings of the 2012 IEEE 9th International Conference on Fuzzy Systems and Knowledge Discovery, 2012, pp. 2174-2177. 
[18] C.Y. Fu, Z. Jiang, W. Wei, A. Wei, “An energy balanced algorithm of LEACH protocol in WSN," IJCSI International Journal of Computer Science, vol. 10, issue 1, pp. 354359, January 2013.

[19] M. J. Handy, M. Haase, D. Timmermann, "Low energy adaptive clustering hierarchy with deterministic cluster head selection," Proceedings of the 4th International Workshop on Mobile and Wireless Communications Network, 2002, pp. 368-372.

[20] S. Corson, J. Macker, "Mobile Ad-hoc Networking (MANET) - Routing Protocol Performance Issues and Evaluation Considerations," RFC2501, University of Maryland, pp. 1-12, January 1999.

[21] A. Al-Maashri, M. Ould-Khaoua, "Performance analysis of MANET routing protocols in the presence of self-similar traffic," Proceedings of the 31st Annual IEEE Conference on Local Computer Networks, 2006, pp. 801-807.

[22] O. Younis, S. Fahmy, "HEED: A hybrid energy efficient distributed clustering approach for adhoc sensor networks," IEEE Transactions on Mobile Computing, vol. 3, issue 4, pp. 366379, December 2004.

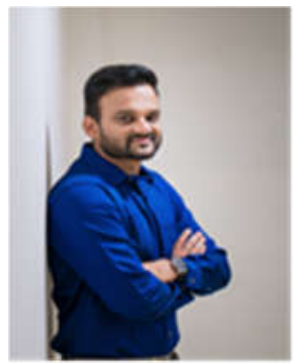

Dr. Rathish Radhakrishnan pursued his Bachelor's degree in the field of Electronics and Communication Engineering at Maharaja Prithvi Engineering College and M.E in VLSI Design from Karpagam University, Coimbatore. He did his doctorate from St. Peter's Institute of Higher Education and Research, Chennai. He published works in the Proceedings of 4 national conferences and published 12 articles. His areas of interests include VLSI Design, Wireless Communication and Networks.

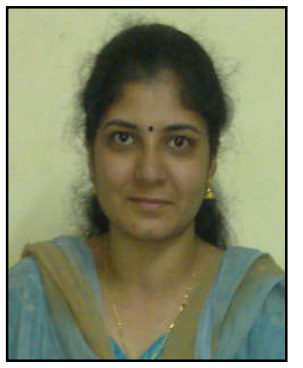

Karpagavadivu Karuppusamy received B.E degree in Computer Science and Engineering at Nandha Engineering College and M.E. in computer science and Engineering at SNS College of Technology, Coimbatore. She published works in the Proceedings of 3 International conferences and in 1 International journal. Her areas of interests are: Networks, Data Warehousing and Data Mining. 